\title{
Le genre Diadesmis (Bacillariophyta) dans les archipels de Crozet et de Kerguelen avec la description de cinq espèces nouvelles
}

\author{
R. Le Cohu ${ }^{1}$ \\ B. Van de Vijver ${ }^{2}$
}

Mots-clés : Diatomées, Diadesmis, nouvelles espèces, subantarctique, Kerguelen, Crozet.

Des investigations portant sur le genre Diadesmis (Bacillariophycées), observé à l'aide du microscope photonique et du microscope électronique à balayage, ont été réalisées dans différents biotopes des archipels de Crozet et de Kerguelen : lacs, mares temporaires, mousses et sols. Dix espèces ont été recensées. Diadesmis arcuata Heiden, décrite à l'origine dans l'archipel de Kerguelen, est réexaminée et typifiée. Cinq espèces nouvelles pour la science sont décrites : Diadesmis comperei, Diadesmis costei, Diadesmis crozetikerguelensis, Diadesmis langebertalotii et Diadesmis subantarctica. Les espèces appartenant au sous-genre Paradiadesmis ont une structure particulière : les interstries sont constituées de tubes creux quadrangulaires.

The diatom genus Diadesmis in the Crozet and Kerguelen Archipelagos with the description of five new species

Keywords : Diatoms, Diadesmis, subantarctic, Kerguelen, Crozet.

The genus Diadesmis (Bacillariophyta) in the Crozet and Kerguelen Archipelagos was studied using light and scanning electron microscopy. Samples were collected from different habitats : lakes, temporary peat-pools, mosses and soils. Ten species have been recorded. The typification of the species Diadesmis arcuata Heiden, described from Kerguelen Archipelago, is reviewed. Five new species are described : Diadesmis comperei, Diadesmis costei, Diadesmis crozetikerguelensis, Diadesmis langebertalotii and Diadesmis subantarctica. The subgenus Paradiadesmis appears to have an unusual structure : the interstriae are quadrangular hollow tubes.

\section{Introduction}

Les archipels de Crozet et de Kerguelen appartiennent à la province biogéographique dite «subantarctique» (Stonehouse 1982). La flore diatomique de ces deux archipels commence à être bien connue (Germain 1937, Bourrelly \& Manguin 1954, Lange-Bertalot \&

1. Centre d'Ecologie des Systèmes Aquatiques Continentaux, UMR C5576, Université Paul Sabatier, 118, route de Narbonne, 31062 Toulouse Cedex 04, France.

2. Université d'Anvers (RUCA), Ecologie polaire, limnologie et paléobiologie, Groenenborgelaan 171 B 2020 Anvers, Belgique.
Le Cohu 1985, Le Cohu 1982, 1983, 1988, 1999, Le Cohu \& Maillard 1983, 1986, Pierre 1977, Vàn de Vijver \& Beyens 1998, 1999 a et b, Van de Vijver et al. 1998, Van de Vijver et al. 2000). Différents biotopes ont été prospectés : lacs, mares temporaires, rivières, mousses et sols. Dans ce contexte, les espèces appartenant au genre Diadesmis (Round et al. 1990) ont été généralement recensées dans le genre Navicula, à l'exception de D. contenta (Grun.) D.G. Mann (Van de Vijver \& Beyens 1998) et de $D$. contenta fo. undulata (Manguin) Van de Vijver \& Beyens (1999 a) qui n'est pas vraisemblablement le bon taxon, l'identification ayant été effectuée à l'aide du seul microscope photonique. 
Lors de sa résurrection (Round et al. 1990), le genre Diadesmis regroupait seulement 7 espèces. Ces dernières années, 14 nouvelles espèces ont été décrites et 7 nouvelles combinaisons proposées (Moser et al. 1998, Lange-Bertalot \& Genkal 1999, Rumrich et al. 2000 et Lange-Bertalot \& Werum 2001), ce qui porte à environ 28 le nombre d'espèces connues dans ce genre. Le genre Diadesmis renferme généralement des taxons de petite taille ; au microscope photonique, certains caractères permettant de discriminer les espèces ne sont pas discernés ; c'est le cas, par exemple, de la structure en $\mathrm{T}$ des terminaisons du raphé. Chez une même espèce, d'un individu à l'autre, les stries peuvent être résolues (la plupart du temps en utilisant un éclairage oblique) ou non. Très souvent, c'est l'examen préalable au microscope électronique à balayage qui permet ensuite de repérer les espèces au microscope photonique.

Le but de ce travail est d'une part, de décrire 5 espèces nouvelles pour la science rencontrées à la fois dans les archipels de Crozet et de Kerguelen et d'autre part, de dresser un premier inventaire des espèces du genre Diadesmis dans les deux archipels. Il faut remarquer que toutes les espèces recensées appartiennent au sous-genre Paradiadesmis Lange-Bertalot \& Le Cohu (Rumrich et al. 2000). L'espèce type du genre Diadesmis, $D$. confervacea Kütz, se caractérise, entre autres, par des stries constituées de pores et atteignant le bord de la valve ; le sous-genre Paradiadesmis présente des stries sous forme de fentes plus ou moins allongées bordées en vue externe par une aire hyaline qui occupe toute la périphérie de la valve. En vue interne, les stries sont séparées en deux parties par une ligne longitudinale latérale marquée par de minuscules saillies sur les interstries. Sur le manteau, les stries sont des pores allongés comme chez $D$. confervacea. Les principaux critères utilisés pour discriminer les espèces ont été les suivants : morphologie générale de la valve, nombre de stries et orientation de celles-ci, largeur de l'aire axiale, largeur de l'aire hyaline latérale, terminaisons du raphé en $\mathrm{T}$ ou non et position du pore distal du raphé.

\section{Matériel et méthodes}

\subsection{Description des sites et modes de prélèvement}

Dans l'archipel de Kerguelen, plusieurs lacs et mares temporaires ont été prospectés. Le lac des Korrigans, le plus étudié lors de cette étude, se situe dans la presqu'île du Gauss à $2,5 \mathrm{~km}$ de la mer mais aucune donnée physico-chimique n'est disponible pour ce lac. Le lac de Marville, localisé au S.E. de la Grande Ile, est séparé de la mer par un cordon littoral et est relié à celle-ci par un canal. Les trois lacs du Val Studer (lac Supérieur, lac des Truites et lac des Saumons) communiquent entre eux et occupent une vallée au N.O. de la base de Port aux Français à 8 km de la mer. Le lac Alicia, bien protégé des vents, se trouve sur l'île Australia dans le golfe du Morbihan à une centaine de mètres de la mer.

Les valeurs de la conductivité et du $\mathrm{pH}$ varient d'un lac à l'autre : le lac Alicia : $245 \mu \mathrm{S} / \mathrm{cm}, 7$; les lacs du Val Studer : 65 - $70 \mu \mathrm{S} / \mathrm{cm}, 5,9$ - 6, 5 ; le lac de Marville : $139 \mu \mathrm{S} / \mathrm{cm}, 6,5$. Les concentrations en azote nitrique sont basses, de l'ordre de 30 à $230 \mu \mathrm{g} / \mathrm{l}$. Les teneurs en phosphore total et phosphore réactif soluble sont faibles, de $32 \mu \mathrm{g} / \mathrm{l}$ dans les lacs du Val Studer à $320 \mu \mathrm{g} / \mathrm{l}$ dans le lac de Marville pour le premier, de $1 \mu \mathrm{g} / \mathrm{l}$ à $3 \mu \mathrm{g} / \mathrm{l}$ pour le second. Les concentrations en silice oscillent de 0,2 à $1 \mathrm{mg} / \mathrm{l}$. Les chlorures varient en fonction de la proximité de la mer, de $11 \mathrm{mg} / \mathrm{l}$ dans les lacs du Val Studer à $30 \mathrm{mg} / \mathrm{l}$ dans le lac de Marville. Les teneurs en $\mathrm{Na}, \mathrm{K}, \mathrm{Ca}$ et $\mathrm{Mg}$ sont toujours inférieures à $5 \mathrm{mg} / \mathrm{l}$. Toutes ces valeurs ont été relevées au cours du printemps 1999.

Les mares temporaires prospectées, tapissées de mousses, sont localisées à environ $1 \mathrm{~km}$ à l'ouest de la base du Port aux Français et présentent une conductivité de l'ordre de $150-160 \mu \mathrm{S} / \mathrm{cm}$ et un $\mathrm{pH}$ légèrement acide. Dans tous les milieux prospectés les échantillons ont été collectés par grattage de galets ou par expression de plantes.

Dans l'île de la Possession (archipel de Crozet), les échantillons proviennent d'expressions de mousses et de différents types de sols. Dans ce dernier cas, le sol sous la végétation était prélevé à l'aide d'une bouteille en PVC de $50 \mathrm{ml}$. Que ce soit dans l'archipel de Kerguelen ou dans l'île de la Possession, les récoltes ont été fixées dans du formol à $5 \%$.

\subsection{Préparation des échantillons}

Les échantillons provenant de l'archipel de Kerguelen ont été traités à l'eau oxygénée 130 volumes, ceux de l'île de la Possession à l'eau oxygénée et au permanganate de potassium (Van der Werff 1955). Pour les observations au microscope photonique (MP), les diatomées, déposées sur une lamelle, ont été séchées puis montées dans la résine synthétique Naphrax. Les photographies ont été réalisées en quasi-totalité avec une caméra JVC 3 CCD KY - F58. Pour les observations au microscope à balayage (MEB), dans le cas des échantillons de l'archipel de Kerguelen, les diatomées nettoyées étaient déposées sur une lamelle fixée au support par du vernis à ongle. Le matériel était métal- 
lisé à l'or palladium et examiné avec un microscope Cambridge Stereoscam $250 \mathrm{MK} 3$ (gamme de voltages utilisés : $10-21 \mathrm{kv}$ ). Dans le cas des échantillons de l'île de la Possession, une partie aliquote de suspension était filtrée à travers un filtre nuclépore à $3 \mu \mathrm{m}$ de maille, une portion du filtre étant fixée au support après séchage à l'air. Le matériel, après métallisation, était observé avec un microscope Philips 515 à 20 kv.

\section{Description des espèces}

\section{Diadesmis arcuata (Heiden) Lange-Bertalot \\ Synonymie : Navicula arcuata Heiden}

Cette espèce dulçaquicole a été décrite dans les sédiments de la baie de l'Observatoire de la Grande Ile de Kerguelen (Heiden \& Kolbe 1928).

\section{Observations}

MP - Typiquement (pl. 33, Fig. 80 Heiden \& Kolbe 1928), les valves sont linéaires, tumides dans la partie médiane, resserrées puis largement subcapitées, arrondies aux extrémités (Figs 1, 2, 3, 4). Cependant, des variations morphologiques sont observées avec une atténuation de ces différents caractères (Figs 5,6,7) et notamment une évolution vers des formes linéaires quadrangulaires chez les petits spécimens (Fig. 8). La longueur varie de 9 à $29 \mu \mathrm{m}$, la largeur de 3,5 à $6 \mu \mathrm{m}$. Ce qui caractérise cette espèce et permet de la distinguer aisément d'autres espèces morphologiquement semblables, c'est l'aire axiale très large qui s'évase au niveau de l'aire centrale et se referme vers les extrémités. En vue typiquement externe (Fig. 8), les stries sont courtes et bordées latéralement par une aire hyaline. Elles sont plus ou moins radiantes dans la partie médiane, subparallèles à parallèles sur leur plus grande longeur (Figs 1, 2), le plus souvent 29 - 32 en $10 \mu \mathrm{m}$, rarement jusqu'à 34. En vue interne (cf MEB), les tries sont interrompues par une ligne longitudinale latérale (Figs 1, 2, 4, têtes de flèches). Le nombre de stries est inférieur à celui dénombré par Heiden (Heiden \& Kolbe 1928) et par Manguin (Bourrelly \& Manguin 1954) ; ces deux auteurs indiquent 35 stries environ. Les deux branches du raphé sont rectilignes, dépourvues de fissures terminales et les pores distaux se situent normalement au niveau des dernières stries.

MEB - La structure des stries est typique du sousgenre Paradiadesmis.

Vue externe (Figs 9, 10, 11, 12).

Les stries courtes (de longueur jamais supérieure à $0,5 \mu \mathrm{m}$ ) et en forme de pores allongés occupent une petite partie de la face valvaire ; elles sont interrompues par une aire hyaline périphérique de largeur supé- rieure à la longueur des stries. Celles-ci reprennent sur le manteau sur tout le pourtour de la valve. Il faut noter que par rapport à ce que l'on observe au MP, les dernières stries peuvent être légèrement convergentes vers les pôles (Figs 9,12). L'aire axiale, toujoursde largeur supérieure à l'aire hyaline latérale, couvre au moins $1 / 3$ de la largeur de la valve et se rétrécit aux extrémités, ce qui est déjà observable au MP ; elle s'élargit en une aire centrale plus ou moins elliptique. Les branches du raphé aux extrémités à pėine marquées sont rectilignes; les pores distaux se situent au niveau des dernières stries.

\section{Vue interne}

Une ligne longitudinale constituée de minuscules crêtes sur les interstries (Figs 13, 14, têtes de flèches) s'étire latéralement sur presque toute la longueur de la valve (Fig. 15). Du côté du sternum, les stries présentent une portion élargie (Figs 15, 16) qui correspond à la partie visible extérieurement sur la face valvaire. La présence d'un hymen, tel que montré par Round et al. (1990, Fig. h, i), n'a jamais été décelée, sans doute érodé par le traitement à l'eau oxygénée. Le nodule central est bombé (Figs 13, 16), en forme de demie sphère, tel que l'a représenté Manguin (Bourrelly \& Manguin 1954). Les pores centraux présentent une ébauche de T (Fig. 16).

Remarques : La figure 10 (pl. 62) de Simonsen (1992) ne représente pas $D$. arcuata mais $D$. coste $i$ nov: spec. La figure (Taf. 83, Fig. 6) présentée par Rumrich et al. (2000) ne peut être rangée dans $D$. arcuata étant donné la différence dans la structure du raphé (extrémités distales en forme de $\mathrm{V}$ très évasé).

Ecologie: Dans l'île de la Possession (archipel de Crozet), D. arcuata est rare dans les sols secs avec une faible conductivité ainsi que dans les mousses. Dans l'archipel de Kerguelen, cette espèce est toujours présente mais jamais abondante ; elle a été recensée dans le périphyton des lacs étudiés ainsi que dans les mares temporaires.

\section{Diadesmis comperei Le Cohu \& Van de Vijver nov. spec.}

\section{MP- Diagnosis}

Valvae lineares leviter tumidae in media parte apicibus rondundatis, 6-15 longae, 2-3 $\mu \mathrm{m}$ latae. Rami raphis filiformi recti. Striae parallelae in media parte, deinde leviter radiantes, subparallelae et in fine vix convergentes ad polos versus, 30-34 striae in $10 \mu \mathrm{m}$. Area axialis bene terminata. Area centralis dilatata generaliter marginata striis curtissimis. 
MEB - Aspectus externus - Striae marginatae lateraliter area hyalina angustissima, minus $1 \mu \mathrm{m}$ latitudinis. Area axialis latitudine constanti in maxima longitudine, incipiens se dilatare ultra centrales poros in area centrale plus minusve elliptica adtingente paene margines valvae et marginata ab 3 ad 5 strias curtissimas. Rami raphis recti filiformi saepe marginati crusta silicis in parte poli cum poris minime expressis et extremis terminalibus in aequo aut vix ultra ultimas strias.

\section{MP - Diagnose}

Valves linéaires aux extrémités arrondies légèrement tumides dans la partie médiane : longueur 6-15 $\mu \mathrm{m}$, largeur $2-3 \mu \mathrm{m}$. Branches du raphé rectilignes filiformes. Stries parallèles dans la partie médiane, ensuite faiblement radiantes, subparallèles et enfin légèrement convergentes vers les pôles, 30-34 en $10 \mu \mathrm{m}$. Aire axiale bien délimitée. Aire centrale élargie bordée par des stries très écourtées.

MEB - Vue externe - Stries bordées latéralement par une aire hyaline très étroite, moins de $1 \mu \mathrm{m}$ de large. Aire axiale de largeur constante sur sa plus grande longeur commençant à s'élargir au delà des pores centraux en une aire centrale plus ou moins elliptique atteignant presque les bords de la valve et bordée par 3 à 5 stries très écourtées. Branches du raphé rectilignes filiformes souvent encadrées par un encroûtement siliceux dans la partie polaire ; pores peu distincts avec des extrémités distales au niveau ou légèrement au delà des dernières stries.

Holotype : Préparation Kerguelen - Korrigans $n^{\circ} 42$. Collection R. Le Cohu, CESAC, Université Paul Sabatier, Toulouse, France.

Localité type : lac des Korrigans - Presqu'île du Gauss - Kerguelen.

Cette espèce peut être également examinée sur la préparation PLP - 016 D. (île de la Possession) Collection Van de Vijver, University of Antwerpen (RUCA), Belgique.

\section{Observations}

MP - Les valves linéaires sont légèrement renflées dans la partie médiane et se terminent par des extrémités arrondies (Figs 17, 18, 19, 20) ; dans quelques cas, le renflement médian est plus accentué (Fig. 21). L'aire axiale est toujours bien délimitée. Des stries parallèles (Fig. 17) bordent l'aire centrale plus ou moins arrondie ; ces stries sont ensuite légèrement radiantes, subparallèles et enfin faiblement convergentes vers les pôles (Figs 17, 19). Les stries paraissent atteindre les bord's de la valve étant donné l'étroitesse de l'aire hyaline marginale (cf: MEB). Les branches du raphé sont rectilignes et filiformes.

MEB - En vue externe, une aire hyaline marginale très étroite, moins de $1 \mu \mathrm{m}$ de large, délimite les stries qui re- prennent sur le manteau (Figs 22, 23, 24). L'aire axiale, de largeur constante sur la plus grande longueur (Figs 22, 23), commence à s'élargir au delà des pores centraux du raphé en une aire centrale plus ou moins elliptique atteignant presque les bords de la valve. Des stries très écourtées (4 en général) délimitent l'aire centrale. Les branches du raphé, rectilignes, présentent le plus souvent un encroûtement siliceux dans la partie distale (Figs 22, 23, flèches) ; les terminaisons sont peu prononcées avec des pores: distaux légèrement au delà des dernières stries. Il faut noter que certains individus présentent des indentations marginales sur les interstries (Fig. 23). En vue interne, la ligne longitudinale de minuscules crête's sur les interstries (Fig. 25, flèche) est très marginale, ce qui confirme les observations en vue externe.

Remarques : $D$. comperei peut se comparer à $D$. templiniana Lange-Bertalot \& Rumrich décrite dans les Andes (Rumrich et al. 2000) ; elle s'en distingue par la partie médiane plus renflée, par la forme de l'aire centrale et par le nombre et l'orientation de stries. Elle présente une ressemblance assez lointaine avec $D$. implicata Lange-Bertalot, Moser \& Metzeltin (Moser et al. 1998) décrite en Nouvelle-Calédonie mais elle s'en différencie nettement par sa morphologie plus élancée, par la partie médiane de la valve moins renflée, par la configuration de l'aire centrale et par le nombre de stries.

Ecologie : Dans l'île de la Possession, D. comperei est assez commune mais en faible abondance dans les sols. Elle semble préférer les sols secs au $\mathrm{pH}$ voisin de la neutralité avec une faible conductivité et de très faible teneurs en azote et phosphore. Dans l'archipel de Kerguelen, cette espèce est rare bien que toujours présente dans les milieux étudiés.

\section{Diadesmis costei Le Cohu \& Van de Vijver nov. spec.}

\section{Diagnosis}

MP - Valvae lineares, tumidae in media parte, angustatae deinde late subcapitatae rotundatae ad apices. Longitudino : $12-15 \mu \mathrm{m}$, latitudo : $4-5 \mu \mathrm{m}$. Striae leviter radiantes, subparallelae in maxima longitudine deinde leviter convergentes ad polos versus, solum discernandae cum illuminatione obliqua, 34-40 in $10 \mu \mathrm{m}$. Area axialis angusta dilatata in media parte in area centrale plus minusve elliptica. Rami raphis filiformi recti cum extremitatibus generaliter citra ultimas strias.

MEB - Aspectus externus - Striae curtae, quaeque minus $1 / 10$ latitudinis valvae. Striae in limbo tenentes totum ambitum valvae. Area centralis dilatata marginata generaliter 6 striis curtissimis. Area axialis se dilatans ad polos. Area hyalina lateralis tenens majorem superficiem quam striae. Rami raphis recti cum poris centralibus parum aut minime dilatatis et extremitatibus terminalibus generaliter leviter citra ultimas strias.

\section{Diagnose}

MP - Valves linéaires, tumides dans la partie médiane, resserrées puis largement subcapitatées arrondies 


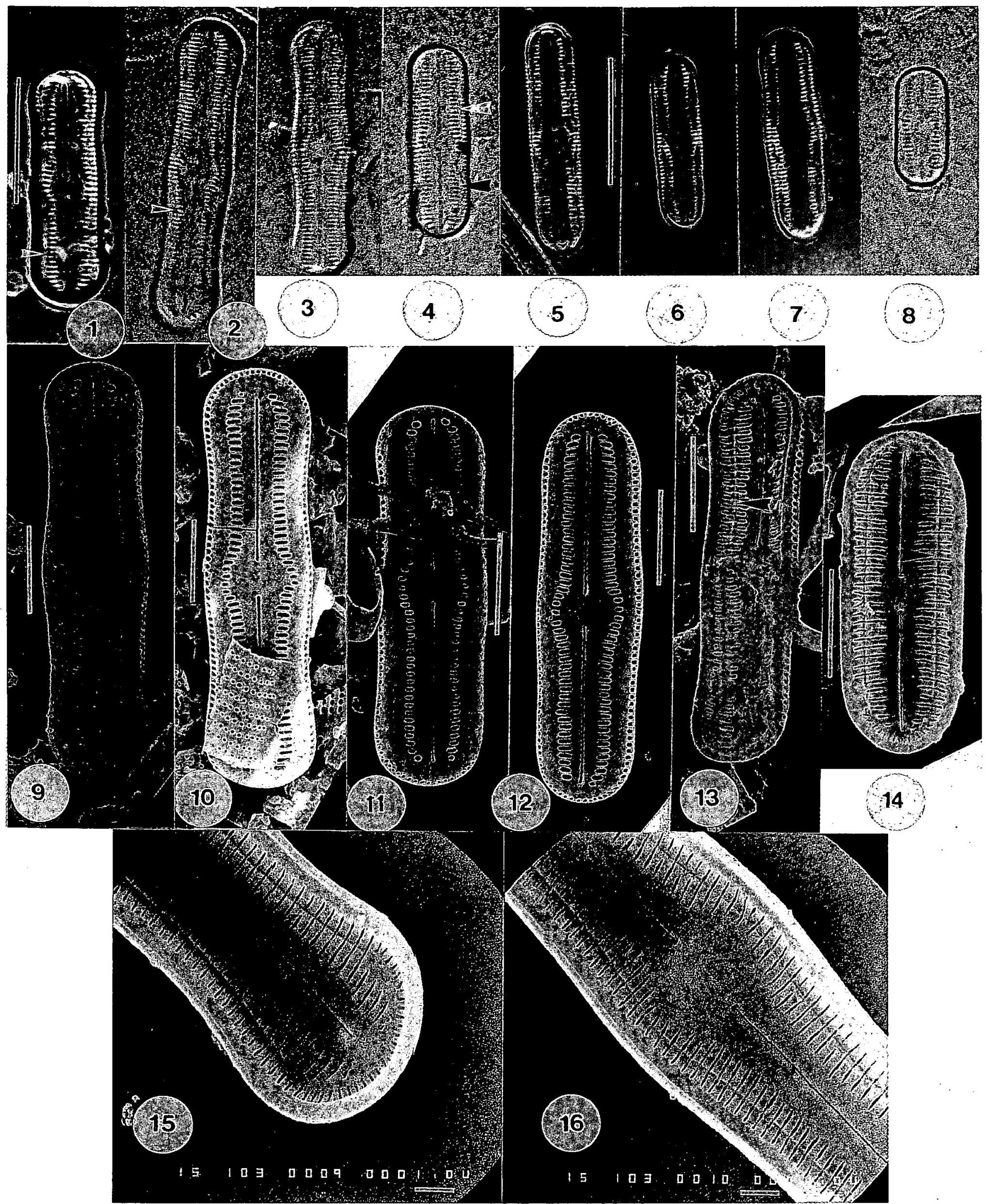

Planche 1. Diadesmis arcuata. MP : Figs 1, 2, 3, 4, 5, 6, 7, 8 (Figs 1, 2,.4, têtes de flèches indiquant la ligne longitudinale interne). MEB, vue externe : Figs $9,10,11,12$; vue interne : Figs 13;14, têtes de flèches indiquant les minuscules crêtes sur les interstries, Figs 15 , 16. Echelles : $10 \mu \mathrm{m}$ (Figs 1, 2, 3, 4, 5, 6, 7, 8); $4 \mu \mathrm{m}$ (Figs 9, 10, 11, 12, 13, 14); $1 \mu \mathrm{m}$ (Figs 15, 16).

Plate 1. Diadesmis arcuata. MP : Figs 1, 2, 3, 4, 5, 6, 7, 8 (Figs 1, 2, 4, with arrowheads showing the internal longitudinal line). MEB, external view : Figs 9, 10,11, 12 ; internal view : Figs 13,14, arrowheads showing the tiny ridges on the interstriae, Figs 15 , 16. Scales : $10 \mu \mathrm{m}$ (Figs 1, 2, 3, 4, 5, 6, 7, 8) ; $4 \mu \mathrm{m}$ (Figs 9, 10, 11, 12, 13, 14) ; $1 \mu \mathrm{m}$ (Figs 15, 16). 
aux extrémités. Longueur : $12-25 \mu \mathrm{m}$; largeur 4-5 $\mu \mathrm{m}$. Stries faiblement radiantes, subparallèles sur leur plus grande longueur puis légèrement convergentes vers les pôles, seulement visibles avec un éclairage oblique, 34-40 en $10 \mu \mathrm{m}$. Aire axiale étroite élargie en une aire centrale plus ou moins elliptique. Branches du raphé rectilignes filiformes aux extrémités distales généralement légèrement en deçà des dernières stries.

MEB - Vue externe. Stries très courtes, chacune moins de $1 / 10$ de la largeur de la valve. Stries sur le manteau occupant toute la périphérie de la valve. Aire centrale élargie bordée généralement de 6 stries très écourtées. Aire axiale s'élargissant vers les pôles. Aire hyaline latérale occupant une plus grande surface que les stries. Branches du raphé rectilignes avec des pores centraux peu ou pas marqués et des extrémités distales généralement en deçà des dernières stries.

Holotype : Préparation Kerguelen-Korrigans $n^{\circ} 42$ Collection Le Cohu, CESAC, Université Paul Sabatier, Toulouse, France.

Localité type : lac des Korrigans - Presqu'île du Gauss - Kerguelen.

Cette espèce peut être également examinée sur la préparation PLP - 015D (île de la Possession - archipel de Crozet) - Collection Van de Vijver, University of Antwerpen (RUCA), Belgique.

\section{Observations}

MP - Les valves sont linéaires, renflées dans la partie médiane, resserrées puis subcapitées arrondies aux extrémités (Figs 26, 27, 28, 29). L'aire axiale, étroite, s'évase vers les pôles (Fig. 27) et s'élargit en une aire centrale plus ou moins elliptique. En vue externe (Fig. 27), les stries sont bordées latéralement par une large aire hyaline où les stries sont visibles quand la mise au point se fait sur la vue interne (Figs 26, 29). En vue externe (Figs 27, 28), les stries sont courtes, radiantes au niveau de l'aire centrale, subparallèles sur leur plus grande longueur et faiblement convergentes vers les pôles. Les branches du raphé sont rectilignes avec des pores distaux légèrement en deçà des dernières stries.

MEB - En vue externe, particulièrement sur les sujets non érodés (Fig. 30), les stries occupent moins de 1/10 de la largeur des valves. Comme cela a déjà été observé au MP, au niveau de l'aire centrale, les stries très écourtées (généralement 6) sont radiantes ; elles sont ensuite subparallèles avant de devenir convergentes là où l'aire axiale s'évase, l'élargissement étant plus ou moins prononcé suivant les individus (Figs 30, 31, 32). L'aire hyaline marginale est de largeur sensiblement équivalente à celle de l'aire axiale. Les branches du raphé sont rectilignes avec des pores peu ou pas marqués, les extrémités distales se situant légèrement en deçà des dernières stries. En vue interne, l'élargissement de l'aire axiale au voisinage des pôles ainsi que la position de l'hélictoglosse en deçà des dernières stries sont clairement mis en évidence, la position de l'hélictoglosse pouvant être variable en fonction des individus (Figs 33, 34). La ligne longitudinale de minuscules crêtes sur les interstries (Fig. 33, tête de flèche) peut être visible en vue externe sur les sujets très érodés (Fig. 35, têtes de flèches), cette ligne délimitant presque entièrement l'aire hyaline marginale. Les portions de stries élargies en vue interne correspondent aux stries visibles sur la face valvaire en vue externe.

Remarques : $D$. costei est morphologiquement semblable à D. arcuata et souvent de dimensions équivalentes. Au MP, elle s'en distingue surtout par l'étroitesse de son aire axiale mais également par le nombre de stries, l'élargissement de l'aire axiale au voisinage des pôles et la position des extrémités distales du raphé. Au MEB, outre les caractères déjà visibles au MP, $D$. costei se différencie de $D$. arcuata par la largeur de l'aire hyaline marginale, celle-ci étant moins large que l'aire axiale chez la seconde espèce.

Ecologie : Dans l'île de la Possession, D. costei est commune dans les sols relativement secs (40-50\% d'humidité relative), à $\mathrm{pH}$ voisin de la neutralité, avec une faible conductivité et des valeurs en chlorures élevées. Dans l'archipel de Kerguelen, D. costei se rencontre dans tous les milieux étudiés mais son habitat préférentiel reste inconnu.

\section{Diadesmis crozetikerguelensis Le Cohu \& Van de Vijver nov. spec. \\ Diagnosis}

MP - Valvae lineares marginibus leviter undulatis et apicibus subcapitatis rotundatis levissime eadem latitudine ac undulae, in media parte leviter concavae, 10-

Planche II. Diadesmis comperei. MP : Figs 17, 18, 19, 20, 21 - MEB, vue externe : Figs 22, 23, flèches indiquant l'encroûtement siliceux au voisinage des poles, Fig. 24 ; vue interne : Fig 25 , flèche indiquant les minuscules crêtes sur les interstries. D. costei. MP : Figs $26,27,28$. MEB, vue externe : Figs $30,31,32$; vue interne : Fig. 33, tête de flèche indiquant la ligne longitudinale avec de minuscules crêtes sur les interstries, Fig. 34 ; Fig. 35, vue externe d'une valve érodée avec la ligne longitudinale interne visible (têtes de flèches). Echelles : $10 \mu \mathrm{m}$ (Figs 17, 18 , $19,20,21,26,27,28,29,33$ ) ; $4 \mu \mathrm{m}$ (Figs 23, 24, 30, 31, 32, 34, 35) ; $2 \mu \mathrm{m}$ (Figs 22,25).

Plate II. Diadesmis comperei. MP : Figs 17, 18, 19, 20, 21 - MEB, external view : Figs 22, 23, arrows showing the siliceous thickening in the vicinity of the poles, Fig. 24 ; internal wiew : Fig. 25, arrow showing the tiny ridges on the interstriae. D. costei. MP : Figs 26, 27, 28. MEB, external view : Figs $30,31,32$; internal view : Fig. 33, arrowhead showing the longitudinal line of tiny ridges on the interstriae, Fig. 34 ; Fig. 35 , external view of an eroded valve with the internal longitudinal line visible (arrowheads). Scales : $10 \mu \mathrm{m}$ (Figs 17, 18, 19, 20, 21, 26, 27, $28,29,33$ ) ; $4 \mu \mathrm{m}$ (Figs 23, 24, 30, 31, 32, 34, 35) ; $2 \mu \mathrm{m}$ (Figs 22,25). 


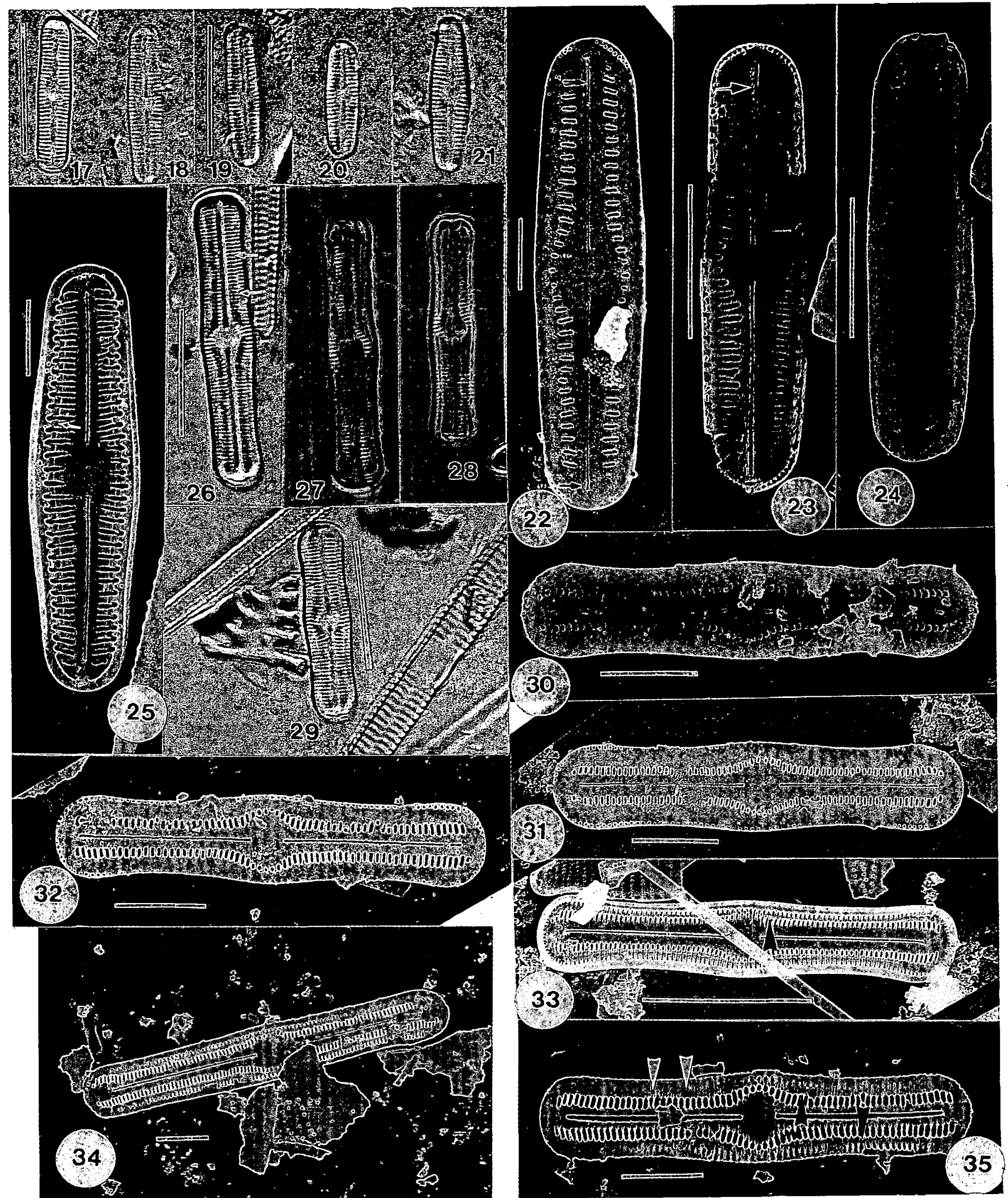


$15 \mu \mathrm{m}$ longae, $2-4 \mu \mathrm{m}$ latae. Striae vix radiantes et in fine leviter convergentes ad polos versus, $30-32 \mu \mathrm{m}$. Rami raphis recti sine poris conspicuis. Area axialis bene terminata non angustata ad polos. Area centralis dilatata communiter marginata 3 striis parallelis.

MEB - Aspectus externus. Rami raphis filiformi cum poris parum notatis et extremis terminalibus desinentibus juxta ultimas strias. Area axialis tenens circiter 1/3 latitudinis valvae, non angustata ad apices. Area hyalina lateralis latitudine majore quam longitudino striarum.

\section{Diagnose}

MP - Valves linéaires à bords faiblement ondulés, légèrement concaves dans la partie médiane, aux extrémités subcapitées arrondies sensiblement de même largeur que les ondulations. Longueur : 10-15 $\mu \mathrm{m}$; largeur : $2-3 \mu \mathrm{m}$. Stries faiblement radiantes puis légèrement convergentes vers les pôles, 30-32 en $10 \mu \mathrm{m}$. Branches du raphé rectilignes aux pores peu ou pas marqués. Aire axiale bien délimitée non resserrée vers les pôles. Aire centrale élargie bordée généralement par 3 stries parallèles.

MEB - Vue externe. Branches du raphé rectilignes filiformes avec des pores peu marqués, extrémités distales se terminant au niveau des dernières stries. Aire axiale occupant environ $1 / 3$ de la largeur de la valve, non resserrée aux extrémités. Aire hyaline latérale de largeur supérieure à la longueur des stries.

Holotype : Préparation Kerguelen-Korrigans $n^{\circ} 42$ Collection Le Cohu, CESAC, Université Paul Sabatier, Toulouse, France.

Localité type : lac des Korrigans - Presqu'île du Gauss, Kerguelen.

Cette espèce peut être également examinée sur la préparation PLP - 014D (île de la Possession - archipel de Crozet) - Collection Van de Vijver, University of Antwerpen (RUCA), Belgique.

\section{Observations}

MP - D. crozetikerguelensis montre une faible variabilité morphologique (Figs. 36, 37, 38, 39). Les valves, concaves dans la partie médiane, présentent une double ondulation peu prononcée de même largeur que les extrémités arrondies. L'aire axiale, de largeur constante, s'arrondit en une aire centrale délimitée par 3 stries (Fig. 38). Les branches du raphé sont rectilignes. Les figures 37 et 38 représentent des vues internes (cf. MEB) avec une ligne longitudinale (flèches) séparant les stries en deux tronçons dont la partie ad aire axiale est seule visible extérieurement. Les figures 36 et 39 représentent des vues externes avec une aire hyaline marginale où les stries sont peu ou pas visibles.

MEB - Vue externe (Fig. 40). Les branches du raphé sont rectilignes avec des pores peu prononcés et des extrémités distales très légèrement au delà des dernières stries. L'aire axiale, de largeur constante, occupe plus du 1/3 de la largeur de la valve et s'élargit en une aire centrale délimitée par 3 stries. La largeur de l'aire hyaline marginale est inférieure à celle de l'aire axiale et supérieure à la longueur des stries.

Vue interne (Fig. 41). L'organisation des stries est conforme au sous-genre Paradiadesmis avec une ligne longitudinale de minuscules crêtes sur les interstries. La partie brisée (Fig. 40, flèche) montre la structure de la valve qui semble assez particulière ; les interstries sont constituées de tubes creux de section quadrangulaire.

Remarques : $D$. crozetikerguelensis est morphologiquement très proche de $D$. corrugata Lange-Bertalot, Moser \& Metzeltin (Moser et al. 1998) décrite en Nouvelle-Calédonie. Au MP, elle s'en distingue par le nombre de stries et par les extrémités beaucoup moins capitées. Au MEB, la structure du raphé est différente ; chez $D$. corrugata, les extrémités du raphé sont en forme de $\mathrm{T}$.

Ecologie : Dans l'île de la Possession, D. crozetikerguelensis est commune dans les sols avec un taux d'humidité relative de $50 \%$, des valeurs du phosphore, du chlore et de la conductivité supérieures à celles relevées usuellement dans l'île.

Dans l'archipel de Kerguelen, cette espèce a été recensée dans le lac des Korrigans et dans des étangs (Fig. 36 préparation $\mathrm{AD} 8904$, collection Manguin, Museum Paris) ainsi

Planche III. Diadesmis crozetikerguelensis. MP : Figs 36, 37, 38, 39 (Figs 37, 38, flèches indiquant la ligne longitudinale interne). MEB, vue externe : Fig. 40 ; vue externe : Fig. 41, flèche indiquant les interstries creuses. D. langebertalotii. MP : Fig. 42. MEB, vue externe : Figs 43, 44, 45 ; vue interne : Fig. 46, flèche indiquant les minuscules crêtes sur les interstries; Fig. 47, 3 copulae (têtes de flèche). D. subantarctica. MP : Fig. 48, 49, 50, 51, 52. MEB, vue externe : Fig. 53. Echelles : $10 \mu \mathrm{m}$ (Figs 36, 37, 38, 39, 42, 48, 49, 50, 51, 52); $4 \mu \mathrm{m}$ (Figs 40, 41, 43, 45, $46,47,53) ; 2 \mu \mathrm{m}$ (Fig. 44).

Plate III. Diadesmis crozetikerguelensis. MP : Figs 36, 37, 38, 39 (Figs 37, 38, arrows showing the internal longitudinal line). MEB, external view : Fig. 40 ; internal view : Fig. 41, arrow showing the hollow interstriae. D. langebertalotii. MP : Fig. 42. MEB, external view : Figs 43, 44, 45 ; internal view : Fig. 46, arrow showing the tiny ridges on the interstriae; Fig. 47, 3 copulae (arrowheads). D. subantarctica. MP : Fig. 48, 49, 50, 51, 52. MEB, external view : Fig. 53. Scales : $10 \mu \mathrm{m}$ (Figs 36, 37, 38, 39, 42, 48, 49, 50, 51, 52) ; $4 \mu \mathrm{m}$ (Figs 40, 41, 43, 45, 46, 47, 53) ; $2 \mu \mathrm{m}$ (Fig. 44). 


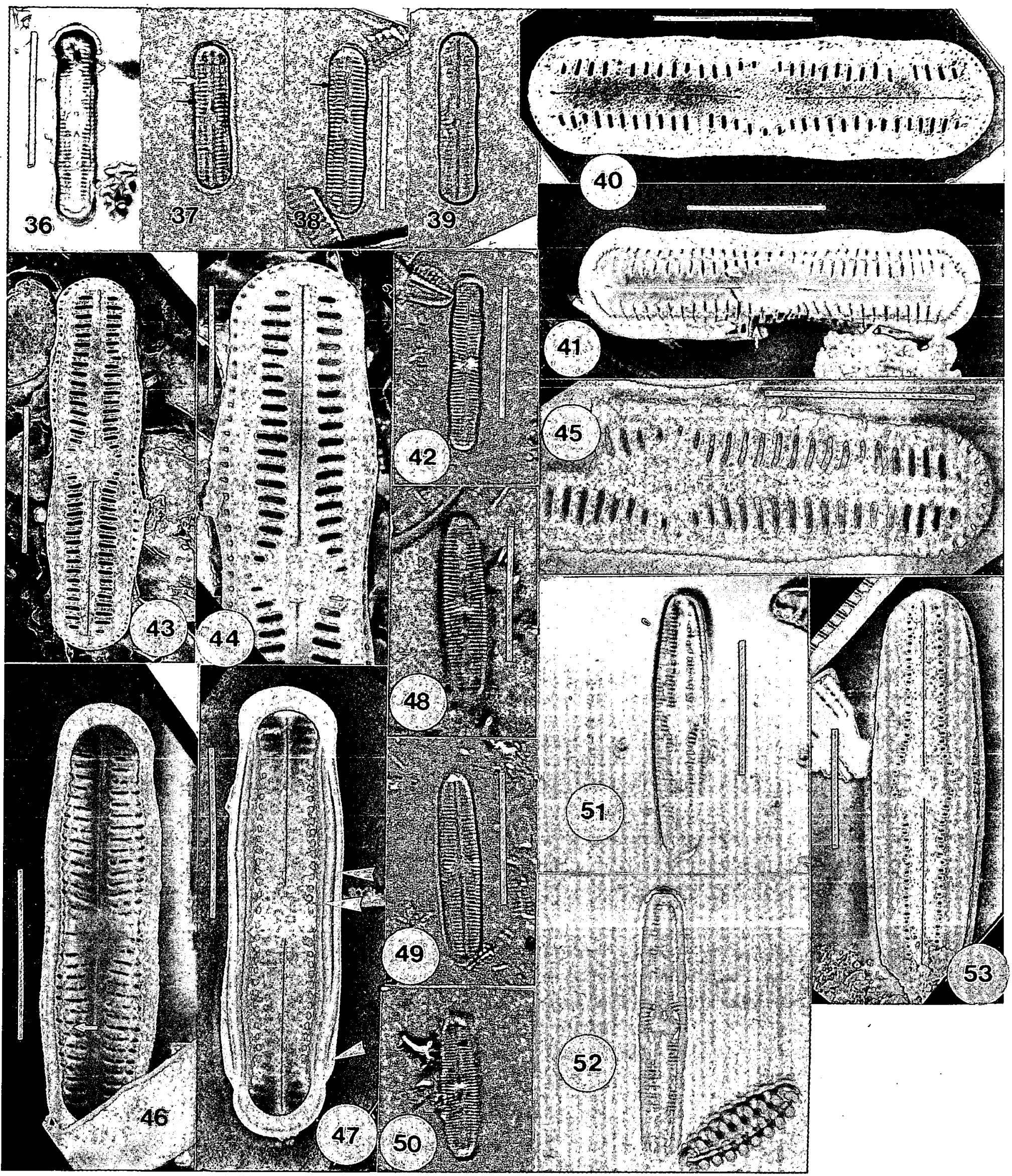


que dans les mares temporaires étudiées. Son écologie reste à préciser.

\section{Diadesmis langebertalotii Van de Vijver \& Le Cohu nov. spec. \\ Diagnosis}

MP - Valvae lineares duplice undula, concavae in media parte, cum apicibus rotundatis latitudine leviter minore quam undulae. Longitudo : 9-13 $\mu \mathrm{m}$, latitudo : 2-3 $\mu \mathrm{m}$. Area axialis angusta. Area centralis plus minusve rotundata posita paene usque ad margines valvae. Striae leviter radiantes in paene longitudine tota, solum discernandae cum illuminatione obliqua, 34-40 in $10 \mu \mathrm{m}$. Rami raphis filiformi recti poris inconspicuis.

MEB - Aspectus externus. Rami raphis recti extremitatibus centralibus et terminalibus in forma litterae $\mathrm{T}$, extremitates terminales leviter ultra ultimas strias. Area axialis latitudine constanti praeter in aequo areae centralis. Area centralis dilatata marginata striis curtissimis quarum quaedum possunt abesse.

\section{Diagnose}

MP - Valves linéaires à double ondulation, concaves dans la partie médiane, aux extrémités arrondies de largeur légèrement inférieure aux ondulations. Longueur : $9-13 \mu \mathrm{m}$, largeur 2-3 $\mu \mathrm{m}$. Aire axiale étroite. Aire centrale plus ou moins arrondie atteignant presque les bords de la valve. Stries faiblement radiantes sur leur plus grande longueur, seulement visibles avec un éclairage oblique, $36-40 \mu \mathrm{m}$. Branches du raphé filiformes rectilignes avec des pores indistincts.

MEB - Vue externe. Branches du raphé rectilignes aux terminaisons en $\mathrm{T}$, extrémités distales légèrement au delà des dernières stries. Aire axiale de largeur constante s'élargissant en une aire centrale bordée de stries écourtées dont certaines peuvent être manquantes.

Holotype : Préparation PLP - 017 D. Collection Van de Vijver, University of Antwerpen (RUCA), Belgique.

Localité type : sols - île de la Possession - Archipel de Crozet.

Cette espèce peut être également examinée sur les préparations $n^{\circ} 41$ et 42 Kerguelen-Korrigans, Collection R. Le Cohu, CESAC, Université P. Sabatier, Toulouse, France.

\section{Observations}

MP - Les valves linéaires à double ondulation, concaves dans la partie médiane, se terminent par des extrémités ar- rondies légèrement subcapitées de largeur légèrement inférieure aux ondulations (Fig. 42). L'aire axiale est étroite, ce qui la différencie de $D$. crozetikerguelensis précédemment décrite (Fig. 38). L'aire centrale atteint presque les bords de la valve alors que, chez $D$. crozetikerguelensis, cette aire centrale est nettement délimitée par trois stries (Fig. 38). Les branches du raphé sont rectilignes avec des pores indistincts. Les stries sont légèrement radiantes sur leur plus grande longueur (Fig. 42).

MEB - En vue externe, les branches du raphé, rectilignes, se terminent en $\mathrm{T}$, les extrémités distales étant légèrement au delà des dernières stries (Fig. 43, 44); en vue interne, les pores proximaux ne sont pas différenciés et les hélictoglosses à peine marqués (Fig: 47). En vue externe, l'aire centrale est bordée d'un nombre variable de stries ( 2 à 4$)$ très écourtées car certaines peuvent être manquantes (Figs 43, 45) ; en fait, seules sont visibles extérieurement les stries qui en vue interne se prolongent au delà de la ligne longitudinale de minuscules crêtes sur les interstries (Fig. 46, flèche) du côté de l'aire axiale. Les stries fermées à l'intérieur (Fig. 47) sont légèrement radiantes jusqu'à l'infléchissement des ondulations pour devenir subparallèles à faiblement convergentes vers les extrémités (Figs 43, 45). 3 copulae ont pu être observées (Fig. 47).

Remarques : Au MP, cette espèce se différencie de $D$. crozetikerguelensis par des ondulations plus prononcées, par les extrémités moins larges que les ondulations, par l'aire centrale atteignant presque les bords de la valve et par le nombre de stries largement supérieur; au MEB, elle s'en distingue par les extrémités du raphé en T. D. langebertalotii est également très proche de $D$. corrugata Lange-Bertalot, Moser \& Metzeltin (Moser et al. 1998) décrite en Nouvelle-Calédonie ; au MP, elle s'en distingue par la morphologie de la valve et notamment par les pôles moins larges que les ondulations ; au MEB, elle en diffère par la forme de l'aire centrale. $D$. langebertalotii peut également faire penser à $D$. bigibba (Hust.) Lange-Bertalot mais elle s'en sépare par les ondulations beaucoup moins accentuées, par le nombre de stries et par l'aire axiale beaucoup moins large et non resserrée aux extrémités (Simonsen 1987, pl. 301, Fig. 29-31).

Ecologie : Dans l'île de la Possession (Archipel de Crozet), cette espèce se rencontre régulièrement en faible abondance dans les sols humides, légèrement acides avec de fortes concentrations en azote et en chlore. Sa présence semble influencée par les apports d'oiseaux (albatros, manchots). Dans l'archipel de Kerguelen, elle est rare, mais a été rencontrée dans tous les milieux étudiés.

Diadesmis subantarctica Le Cohu \& Van de Vijver nov. spec.

\section{Diagnosis}

MP - Valvae lineares - ellipticae modice inflatae in media parte deinde se tenuentes paulatim usque ad polos rotundatos, 6-20 longae, 2-4 $\mu \mathrm{m}$ latae. Rami raphis filiformi recti. Area axialis angusta. Area centralis latior quam longior marginata 4-5 striis. Striae leviter ra- 
diantes in maxima longitudine, vix convergentes ad vicinitatem polorum, solum discernandae cum illuminatione obliqua; $34-38$ in $10 \mu \mathrm{m}$ aut plus in $10 \mu \mathrm{m}$.

MEB - Aspectus externus - Rami raphis filiformi recti extremis centralibus et terminalibus cum poris parum dilatatis. Extremitates terminales raphis finem habens juxta ultimas strias aut leviter ultra. Striae curtae occupantes minus circiter 1/10 latitudinis valvae, marginata lateraliter area hyalina lata. Area axialis leviter constricta ad polos juxta 2 aut 3 ultimas strias.

\section{Diagnose}

MP - Valves linéaires - elliptiques, modérément élargies dans la partie médiane puis s'amincissant progressivement jusqu'aux extrémités arrondies ; longeur : 6-22 $\mu \mathrm{m}$, largeur : $2-4 \mu \mathrm{m}$. Branches du raphé filiformes rectilignes. Aire axiale étroite. Aire centrale plus large que longue délimitée par 4-5 stries. Stries légèrement radiantes sur leur plus grande longueur, faiblement convergentes au voisinage des pôles, seulement visibles avec un éclairage oblique, $34-38$ ou plus en $10 \mu \mathrm{m}$.

MEB - Vue externe - Branches du raphé rectilignes avec des pores à peine marqués, extrémités distales au niveau ou légèrement au delà des dernières stries. Stries courtes, de longueur inférieure au 1/10 de la largeur de la valve, délimitée latéralement par une aire hyaline large. Aire axiale légèrement resserrée vers les pôles au niveau des 2 ou 3 dernières stries.

Holotype : Préparation Kerguelen-Korrigans $n^{\circ} 41$. collection R. Le Cohu, CESAC, Université P. Sabatier, Toulouse, France.

Localité type : lac des Korrigans - Presqu'île du Gauss - Kerguelen.

Cette espèce peut être également examinée sur la préparation PLP - 018 D. Collection Van de Vijver, University of Antwerpen (RUCA), Belgique.

\section{Observations}

MP - Les valves linéaires - elliptiques sont élargies dans la partie médiane puis s'amincissent progressivement jusqu'aux extrémités arrondies (Figs 48, 49, 50). Les stries sont radiantes sur leur plus grande longueur. Il faut remarquer que les vues internes et les vues externes montrent des différences (cf. MEB). D'une part, l'aire axiale est plus étroite en vue interne (Figs 48,49 ) qu'en vue externe (Figs 51,52 ) et d'autre part, l'aire marginale hyaline est seulement visible en vue externe (Figs 51, 52).

MEB - En vue externe, en fonction de l'érosion due au traitement à l'eau oxygénée, les stries sont plus ou moins courtes mais toujours d'une longueur inférieure au 1/10 de la largeur de la valve (Figs 53, 54, 55). Les branches du raphé sont rectilignes avec des pores peu marqués, les extrémités distales se situant au niveau des dernières stries ou légèrement au delà (Fig. 54). L'aire axiale a tendance à se resserrer légèrement au niveau des extrémités distales du raphé (Figs $53,55)$; elle commence à s'élargir en une aire centrale plus ou moins elliptique en deçà des pores centraux du raphé. Les stries, délimitées par une aire hyaline marginale large, sont radiantes sur leur plus grande longueur et deviennent convergentes au voisinage des pôles.

En vue interne, il faut remarquer que du côté aire axiale, par rapport à la ligne longitudinale de minuscules crêtes sur les interstries (Fig. 56 têtes de flèche), les stries présentent une portion élargie qui correspond aux stries visibles extérieurement ; cette portion élargie se prolonge par un tronçon plus fin (Fig. 56, flèche) fermé intérieurement et extérieurement, ce qui explique pourquoi l'aire axiale est plus étroite en vue interne qu'en vue externe. Ce tronçon plus fin peut plus ou moins disparaître en fonction de l'érosion des valves (Fig. 57).

Remarques : Au MP, D. subantarctica se distingue assez aisément de $D$. comperei par la morphologie générale de la valve, par le nombre de stries et par la forme de l'aire centrale. D. subantarctica peut être rapprochée de D. implicata Lange-Bertalot, Moser \& Metzeltin (Moser et al. 1998) ; au MP, cette dernière se différencie de $D$. subantarctica par la partie médiane beaucoup plus renflée et par la forme de l'aire centrale. Notre espèce présente également une certaine ressemblance avec $D$. templiniana Lange-Bertalot \& Rumrich (Rumrich et al. 2000) ; au MP, elle s'en distingue aisément par le nombre de stries et par la configuration de l'aire centrale.

Ecologie : Dans les îles de Kerguelen, $D$. subantarctica a été recensée dans le lac des Korrigans et dans les lacs du Val Studer où la conductivité est faible, le $\mathrm{pH}$ acide et les teneurs en chlore peu élevées ; cependant, la présence de cette espèce est sporadique.

Dans l'île de la Possession, cette espèce est rare ; elle a été rencontrée dans les sols à taux d'humidité relative voisin de $50 \%$, à pH acide et à des concentrations en chlore relativement élevées.

\section{Diadesmis aff. contenta (Grunow) D.G. Mann}

Au MP (Figs 58,59), ce taxon peut être attribué à $D$. contenta étant donné la morphologie de la valve, les dimensions (longueur : $6-11 \mu \mathrm{m}$; largeur : 2,5-3,5 $\mu \mathrm{m}$ ), le nombre de stries (36-40 in $10 \mu \mathrm{m})$ et la configuration de l'aire centrale. Il se différencie nettement de $D$. paracontenta LangeBertalot \& Werum (Lange-Bertalot \& Genkal 1999) par le nombre de stries. Au MEB, une seule observation en vue externe a pu être réalisée à partir des récoltes des îles Kerguelen (Fig. 60). L'absence de stries au niveau de l'aire centrale et la largeur de l'aire hyaline marginale sont conformes à l'espèce $D$. contenta ; par contre, les terminaisons en $T$ des branches du raphé, typique de cette espèce (cf. Fig. 5, Taf. 28, Moser et al. 1998 ; Fig. 9, p. 531, Round et al. 1990) font défaut. En vue interne, des extrémités proximales «classiques» du raphé (Fig. 61) et des extrémités proximales en T (Fig. 62, difficilement visible, flèche) ont pu être observées. 


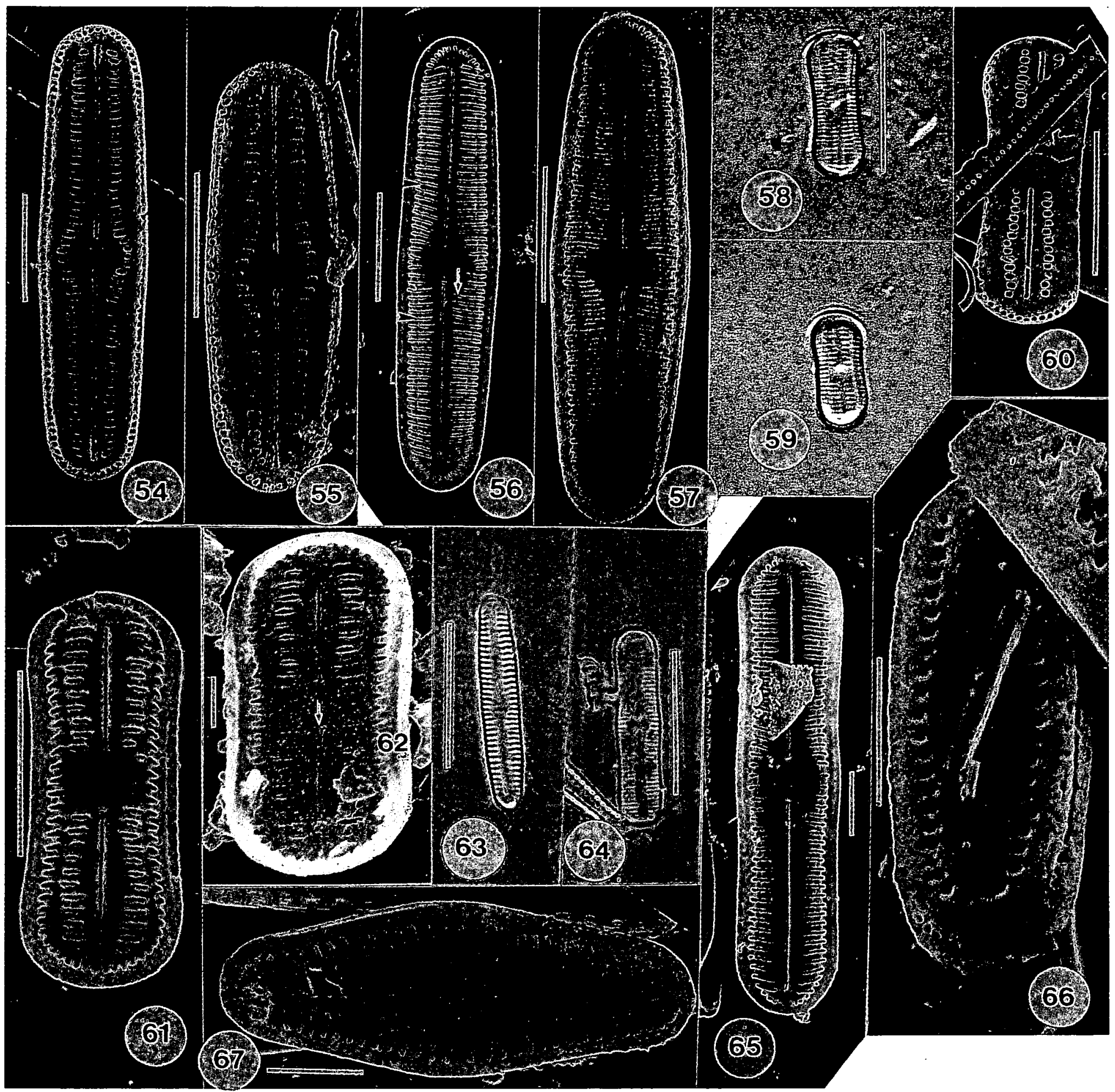

Planche IV. Diadesmis subantarctica. MEB, vue externe : Figs 54, 55 ; vue interne : Fig. 56, têtes de flèche indiquant les minuscules crêtes sur les interstries et flèche indiquant la portion amincie des stries, Fig. 57. D. aff. contenta. MP : Figs 58, 59. MEB, vue externe : Fig. 60 ; vue interne : Figs 61,62 avec flèche indiquant une ébauche de T. D. latestriata. MP : Fig. 63. D. ingeae. MP : Fig. 64. MEB, vue interne : Fig. 65. D. aff. vidalii. MEB, vue externe : Fig. 66. D. aff. perpusilla, vue interne : Fig. 67 . Echelles : $10 \mu \mathrm{m}$ (Figs $58,59,63,64$ ) ; $4 \mu \mathrm{m}$ (Figs $54,55,56,57,60,61$ ) $; 2 \mu \mathrm{m}$ (Figs $65,66,67$ ) ; $1 \mu \mathrm{m}$ (Fig. 62).

Plate IV. Diadesmis subantarctica. MEB, external view : Figs 54,55 ; internal view : Fig. 56, arrowheads showing the tiny ridges on the interstriae and arrow showing the thinner section of the striae, Fig. 57. D. aff. contenta. MP : Figs 58, 59. MEB, external view : Fig. 60 ; internal view : Figs 61, 62 with arrow showing an outline of T. D. latestriata. MP : Fig. 63. D. ingeae. MP : Fig. 64. MEB, internal view : Fig. 65. D. aff. vidalii. MEB, external view : Fig. 66. D. aff. perpusilla, MEB, internal view : Fig. 67 . Scales : $10 \mu \mathrm{m}$ (Figs 58, 59, 63, 64) ; $4 \mu \mathrm{m}$ (Figs 54, 55, 56, 57, 60, 61) ; $2 \mu \mathrm{m}$ (Figs 65, 66, 67) ; $1 \mu \mathrm{m}$ (Fig. 62). 
Il faut remarquer que, dans l'île de la Possession, des individus seulement différenciés par la présence ou l'absence de terminaisons en $\mathrm{T}$ du raphé coexistent. Des études supplémentaires sont donc nécessaires pour déterminer si on a affaire à deux espèces ou si la différence observée est due à une simple variation dans le degré de silicification des valves.

\section{Conclusion}

Cette étude ne prétend pas être un relevé exhaustif de toutes les espèces de Diadesmis pouvant se rencontrer dans les archipels de Crozet et de Kerguelen. Actuellement, 8 espèces nouvelles pour la science peuvent être dénombrées, 5 dans le cadre de ce travail; 3 dans un autre article (Van de Vijver et al., Cryptogamie, Algol., sous presse) consacré à l'île de la Possession (Archipel de Crozet). 10 espèces de Diadesmis sont recensées dans les îles subantarctiques françaises sur environ 38 espèces dans le monde.

Parmi les 3 espèces nouvelles décrites par Van de Vijver et al. (Cryptogamie, Algol., sous presse) dans les sols de l'île de la Possession, D. latestriata (Fig. 63) a été retrouvée au MP dans le lac des Korrigans, mais elle est très rare. Il en est de même de D. ingeae (Figs 64, 65). Dans l'archipel de Kerguelen, d'autres taxons, observés une seule fois au MEB, demandent des investigations complémentaires, tel celui représenté figure 66 (L : $5,7 \mu \mathrm{m} ; 1: 2,1 \mu \mathrm{m}, 40$ stries en $10 \mu \mathrm{m}$ ) proche de D. vidalii (Van de Vijver et al., Cryptogamie, Algol., sous presse) ou tel celui représenté par la figure 67 sans doute voisin de $D$. perpusilla (Grun.) D.G. Mann.

Schoeman \& Archibald (1978) ont fait une revue de tous les taxons inventoriés à l'époque sous le nom de Navicula contenta. Parmi les dessins représentant des spécimens de l'île d'Amsterdam, située au nord de l'archipel de Kerguelen et de climat subtropical, les figures 21 à 29 sont très proches par la morphologie et par le nombre de stries de $D$. crozetikerguelensis; la figure 19 rappelle $D$. subantarctica alors que les figures 28 et 31 peuvent être attribuées à $D$. arcuata. Récemment, Van de Vijver \& Beyens (1999 c) ont d'ailleurs relevé la présence de cette dernière espèce dans cette même île ainsi que celle de $D$. contenta. Les espèces nouvelles décrites dans les îles subantarctiques françaises ont donc sans doute une répartition géographique beaucoup plus vaste.

Par ailleurs, au cours de cette étude, si l'on se réfère à la description du genre Diadesmis (Round et al. 1990), on a observé une seule fois des indentations sur les bords de la valve (Fig. 23). Par contre, le sous-genre Paradiadesmis, tout au moins $D$. crozetikenguelensis, a révélé une structure particulière de la valve où les interstries sont constituées de tubes creux quadrangulaires.

\section{Remerciements}

Cette étude a été réalisée avec le support logistique et financier de l'Institut Français pour la Recherche et la Technologie Polaire (IFRTP) dans le cadre du programme d'écologie terrestre $n^{\circ} 136$ (Dr. Y. Frenot).

\section{Travaux cités}

Bourrelly P. \& Manguin E.. - Contribution à la flore algale d'eau douce des îles Kerguelen. Mem. Inst. Scient. Madagascar, Sér. B : 758.

Germain H. 1937. - Diatomées d'une tourbe de Kerguelen. Bull. Soc. Fr. Microsc., $6: 11-17$.

Heiden H. \& Kolbe R.W. 1928. - Die marinen Diatomeen der Deutschen Südpolar - Expedition 1901-1903. Deutsche Südpolar - Exped. 8 Bot. (5) : 450-715.

Lange-Bertalot H. \& Le Cohu R. 1985. - Raphe like vestiges in the diatom suborder Araphidineae ? Ann. Limnol., 21 : 213-220.

Lange-Bertalot H. \& Genkal S.I. 1999. - Diatomeen aus Sibirien I. Iconographia diatomologica, $6: 292 \mathrm{p}$.

Lange-Bertalot H. \& Werum M. 2001. - Diadesmis fukushimae sp. nov. and some other new or rarely observed taxa of the subgenus Paradiadesmis Lange-Bertalot \& Le Cohu. Diatom, 17 : 3-19.

Le Cohu R. 1982. - Les espèces endémiques de diatomées aux îles Kerguelen. CNRFA, $51: 35-42 /$

Le Cohu R. 1983. - Observations sur deux espèces de diatomées du genre Diatomella : Diatomella hustedtii Manguin et Diatomella ouenkoana Manguin. Cryptogamie, Algol., 4 : 63-71.

Le Cohu R. 1988. - Fragilaria alpestris, Opephora naveana nov. sp. et le complexe Synedra ulna (Bacillariophycées, Araphidinées). Cryptogamie, Algol:, 9 : 101-116.

Le Cohu R. 1999. - Révision des principales espèces de Fragilariales (Bacillariophyta) des îles Kerguelen. Can. J. Bot., 77 : 821-834.

Le Cohu R. \& \& Maillard R. 1983. - Les diatomées monoraphidées des îles Kerguelen. Ann. Limnol., 19 : 143-167.

Le Cohu R. \& Maillard R. 1986. — Diatomées d'eau douce des îles Kerguelen (à l'exclusion des monoraphidées). Ann. Limnol., 22 : 99-118.

Moser G., Lange-Bertalot H. \& Metzeltin D. 1998. — Insel der Endemiten - Geobotanisches Phänomen Neukaledonian. Bibliotheca diatomologica, $38: 464 \mathrm{p}$.

Pierre J.F. 1977. - Les algues des eaux courantes de l'île de la Possession (Archipel de Crozet). Bull. Soc. Phycol., 22 : 79-86.

Round F.E., Crawford R.M. \& Mann D.G. 1990. - The diatoms. Cambridge University Press, Cambridge : $747 \mathrm{p}$.

Rumrich U., Lange-Bertalot H. \& Rumrich M. 2000. - Diatomeen der Anden. Iconographia diatomologica, $9: 673 \mathrm{p}$.

Schoeman F.R. \& Archibald R.E.M. 1978. - The diatom flora of Southern Africa. $N^{\circ}$ 4. C.I.S.R. Special Report Watt 50, Pretoria.

Simonsen R. 1987. - Atlas and catalogue of the diatom types of Friedrich Hustedt. Vol. 1 : Catalogue. Vol. 2 : Atlas, Taf. 1-395. Vol. 3 : Atlas, Taf. 396-772. J. Cramer, Berlin/Stuggart.

Simonsen R. 1992. - The diatom types of Heinrich Heiden in Heiden \& Kolbe 1928. Bibliotheca diatomologica, $24: 1-99+86 \mathrm{pl}$.

Stonehouse B. 1982. - La zonation écologique sous les hautes latitudes australes. CNFRA, $51: 532-537$.

Van de Vijver B. \& Beyens L. 1998. - A preliminary study on the soil diatom assemblages from Iles de la Possession (Crozet, Subantarctica). Eur. J. Soil Biol., 34 (3) : 133-141.

Van de Vijver B. \& Beyens L. 1999a. - Freshwater diatoms from Ile de la Possession (Crozet Archipelago, Sub-Antarctica) : an ecological assessment. Polar Biol., 22 : 178-188. 
Van de Vijver B. \& Beyens L. 1999b. - Moss diatom communities from île de la Possession (Crozet, Subantarctica) and their relationship with moisture. Polar Biol., 22 : 219-231.

Van de Vijver B. \& Beyens L. 1999c. - Première contribution à la flore diatomique de l'île d'Amsterdam (Terres australes et antarctiques françaises). Ann. Limnol., $35: 85-94$.

Van de Vijver B., Beyens L., Gloaguen J.C. \& Frenot Y. 1998. — La flore diatomique de quelques sédiments tourbeux holocènes des îles Kerguelen. Ann. Limnol., 34 : 3-11.
Van de Vijver B., Denys L. \& Beyens L. 2000. - Fragilaria husvikensis sp. nov. (Bacillariophyceae), another Fragilaria species with transapical ribs from Subantarctica. Nova Hedwigia, 70 (3-4) : 537-550.

Van de Vijver B., Ledeganck P. \& Beyens L. - Three new Diadesmis taxa from soils on Ile de Possession (Crozet Archipelago, Subantarctica). (Cryptogamie, Algol., sous presse).

Van der Werff A. 1955. - A new method of concentrating and cleaning diatoms and other organisms. Verh. Int. Verein. Theor. Angew. Limnol., 12 : 276-277. 